\title{
SUR LES RELATIONS ALGÉBRIQUES ENTRE LES INTÉGRALES INDÉFINIES.
}

\author{
PAR \\ ALEXANDRE OSTROWSKI
}

ì Bât.E.

1. Le théorème démontré dans cette note se ramène à ceci: les relations algébriques entre les intégrales indéfinies se réduisent toujours à des relations linéaires.

Pour pouvoir donner un énoncé précis de ce théorème nous allons introduire la notion de corps $L$ de fonctions d'une variable $z$.

Un ensemble $R$ des fonctions de $z$ sera appelé un corps $L$ dans un domaine (ouvert) $D$ du plan des $z$, s'il jouit des trois propriétés suivantes:

A) Chaque fonction de $R$ est uniforme et holomorphe dans $D$, sauf au plus dans un ensemble dénombrable de singularités isolées.

B) Si $f(z)$ est une fonction de $R$, sa dérivée $f^{\prime}(z)$ appartient aussi à $R$.

C) L'ensemble $R$ contient toutes les constantes complexes et est un corps, c'est à dire que si $\alpha$ et $\beta \neq 0$ sont deux grandeurs de $R$, les grandeurs $\alpha+\beta$, $\alpha-\beta, \alpha \beta, \frac{\alpha}{\beta}$ appartiennent aussi à $R$.

2. Alors on a le théorème suivant:

Soit $R$ un corps $L$ dans le domaine $D$ du plan des $z$ et soient

$(2, I)$

$$
\varphi_{v}(z) \quad(v=\bar{i}, \ldots, n)
$$

$n$ grandcurs de $\boldsymbol{h}$ et

$(2,2)$

$$
\psi_{v}(z)=\int \varphi_{v}(z) d z \quad(\nu=I, \ldots, n)
$$

leurs intégrales indéfnies. Soit 
un polynôme en $w_{1}, \ldots, w_{n}$ qui ne s'amule pas identiquement et dont les coefficients sont des fonctions de $z$ du corps $R$.

Alors, s'il existe une relation identique

$$
(2,4) \quad \boldsymbol{F}\left(\psi_{1}(z), \ldots, \psi_{n}(z) ; z\right) \equiv 0 \text {, }
$$

il existe aussi une relation identique linéaive

$(2,5)$

$$
\sum_{v=1}^{n} \alpha_{v}, \psi_{v}(z) \equiv \gamma(z)
$$

où $\gamma(z)$ est une fonction de $R$ et où $\alpha_{1}, \ldots, \alpha_{n}$ sont des grandeurs indépendantes de $z$, dont l'une au moins ne s'annule pas.

3. Démonstration. Nous désignons par $P(z)$ le point de l'espace des $n+1$ variables complexes $w_{1}, \ldots, w_{n}, z$, donné par

$$
w_{1}=\psi_{1}(z), \ldots, w_{n}=\psi_{n}(z), z
$$

Désignons par $\bar{F}$ l'ensemble de termes de $(2,3)$ en $w_{1}, \ldots, u_{n}$ dont les dimensions sont maximum. $\bar{F}$ est un polynôme homogène en $w_{1}, \ldots, w_{n}$ de dimension $d$. Nous pouvons évidemment supposer que $F$ soit choisi de manière que $d$ soit aussi petit que possible. Ordonnons les termes de $\bar{F}$ d'après le principe lexicographique et désignons alors le plus haut terme de $\overline{\boldsymbol{F}}$ par

$$
f(z) w_{1}^{v_{1}} \ldots w_{n}^{v_{n}}
$$

Cela veut dire que les exposants de $w_{1}$ dans chaque terme de $\bar{F}$ sont $\leqq \nu_{1}$; dans chaque terme de $\bar{F}$ contenant $w_{1}^{\nu_{1}}$, l'exposant de $w_{2}$ est $\leqq v_{2}$ etc. On peut évidemment diviser $F$ par $f(z)$ de sorte que le premier terme de $\bar{F}$ peut être supposé dès le début de la forme

$$
v_{1}^{v_{1}} \ldots w_{n}^{v}, \quad \nu_{1}+\cdots+\nu_{n}=d
$$

Nous pourons de plus supposer que parmi les plus hauts termes de $\bar{F}$ pour les différents polynômes $F$ satisfaisant à nos conditions, $(3,2)$ est le plus bas.

En différentiant la relation $(2,4)$ on voit que l'expression

$$
\varphi_{1}(z) \frac{\partial F}{\partial w_{1}}+\cdots+\varphi_{n}(z) \frac{\partial F}{\partial w_{n}}+\frac{\partial F}{\partial z}
$$

s'annule en chaque point $P(z), z: D$, où elle est régulière. 
Sur les relations algébriques entre les intégrales indéfinies.

D'autre part, dans le polynôme $(3,4)$ en $w_{1}, \ldots, w_{n}$ les termes de la dimension maximum $d$ sont tous contenus dans $\frac{\partial F}{\partial z}$. Et puisque la dérivée partielle de $(3,3)$ par rapport à $z$ est identiquement nulle, le plus haut parmi les termes de dimension $d$ en $(3,4)$ écrits dans l'ordre lexicographique, doit être plus bas que $(3,3)$. Donc, l'expression $(3,4)$ s'annule identiquement en $w_{1}, \ldots, w_{n}, z$, et nous voyons que notre fonction $F$ satisfait à l'équation différentielle

$$
\varphi_{1}(z) \frac{\partial F}{\partial w_{1}}+\cdots+\varphi_{n}(z) \frac{\partial F}{\partial w_{n}}+\frac{\partial F}{\partial z}=0
$$

4. Or, les $n$ expressions $\varphi_{v} \frac{\partial F}{\partial w_{v}}$ ne contiennent pas de termes de dimension $d$ en $w_{1}, \ldots, w_{n}$. Donc, les termes de dimension $d$ en $\frac{\partial F}{\partial z}$ doirent se détruire, ce qui n'est possible que si les coefficients de ces termes sont indépendants de $z$. Donc $\bar{F}$ est un polynôme en $w_{1}, \ldots, w_{n}$ ì roefficients constants.

Soit $\nu_{k}$ le premier des $n$ exposants $\nu_{1}, \nu_{2}, \ldots, \nu_{u}$, qui est $>0$. Daus ce cas $\bar{F}$ ne dépend que de $w_{k}, w_{k+1}, \ldots, w_{n}$. Rassemblons dans l'expression $(3,4)$ les coefficients de

$$
P=w_{k}^{v k-1} w_{k+1}^{v k+1} \cdots w_{n}^{v n}
$$

Désignons par $-\gamma(z)$ le coefficient de $(4,1)$ en $F$ et soient

$$
\mathrm{I}, \boldsymbol{\beta}_{k+1}, \boldsymbol{\beta}_{k+2}, \ldots, \boldsymbol{\beta}_{n}
$$

les coefficients (constants) des autres termes de $F$ :

$$
w_{k} P, w_{k+1} P, w_{k+2} P, \ldots, w_{n} P
$$

qui engendrent $(4,1)$ par différentiation.

Alors le coefficient total de $P$ dans $(3,4)$ est évidemment égal à

$$
v_{k} \varphi_{k}+\left(v_{k+1}+1\right) \beta_{k+1} \varphi_{k+1}+\cdots+\left(\nu_{n}+1\right) \beta_{n} \varphi_{n}-\gamma^{\prime}(z) .
$$

D'autre part, d'après $(3,5)$, la somme $(4,2)$ s'annule identiquement. Il résulte, en intégrant, la relation

$(4,3) \quad v_{k} \psi_{k}+\left(\nu_{k+1}+1\right) \beta_{k+1} \psi_{k+1}+\cdots+\left(\nu_{n}+1\right) \beta_{n} \psi_{n}=\gamma(z)+$ const.

Or, ici $\gamma$ est l'un des coefficients de $F$, donc une grandeur de $R$. En outre, les coefficients de la relation $(4,3)$ ne sont pas tous $=0$, puisque $v_{k}$ est positif.

Notre théorème est démontré. 
Ce théorème peut d'ailleurs être considérablement généralisé.

En reserrant un peu les hypothèses portant sur les intégrales $(2,2)$ on peut démontrer que l'existence d'une relation $(2,4)$ a pour conséquence l'existence d'une relation linéaire non seulement quand $F$ est un polynôme, mais aussi si $F$ 's'obtient à partir des variables $\dot{w}_{1}, \ldots, w_{n}$ et des grandeurs de $R$ en combinant les signes des fonctions algébriques, du logarithme et de la fonction exponentielle. On peut même admettre, à côté du logarithme et de la fonction exponentielle, les intégrales des fonctions algébriques et leurs fonctions inverses. ${ }^{1}$

La démonstration de ce résultat exige toutefois des développements assez étendus qui sortent du cadre de cette note.

${ }^{1}$ Ce résultat généralise, en le précisant, un énoncé de M. J. F. Ritt (Trans. Am. Math. Soc., 25, 211-222, 1923) d'après lequel une intégrale indéfinie $w$ d'une fonction élémentaire de $z$ au sens de Liouville est elle-même élémentaire au sens de Liouville si elle satisfait à une équation $F\left(w^{\prime}, z\right)=0$, où $F(w, z)$ est une fonction élémentaire au sens de Lionville, c'est-à-dire formée au moyen des signes de fonctions algébriques, du logarithme et de la fonction exponentielle.

Bâle, le 25 juin 1945. 